\title{
A review of fundus autofluorescence imaging
}

\author{
DJ Booysen*
}

PO Box 339, Paardekraal, 1752 South Africa

$<$ dirk@dirkbooysen.co.za $>$

Received 23 March 2012; revised version accepted 25 February 2013

\begin{abstract}
Autofluorescence photography of the retina provides important diagnostic information about diseases that affect the outer retina; more specifically the retinal pigment epithelium and photoreceptors. Fundus autofluorescence can also
\end{abstract}

be used to evaluate macular pigment density and other diseases of the retina and choroid. It is a non-invasive clinical tool which has the potential to revolutionise clinical retina practice. ( $S A f r$ Optom 2013 72(1) 46-53)

Key words: Autofluorescence, lipofuscin, macular pigment, retinal pigment epithelium

\section{Introduction}

Fundus autofluorescence using a confocal scanning laser ophthalmoscope (cSLO) was first described in 1995 by Von Rückmann et al ${ }^{1}$. The cSLO was used to image the distribution of lipofuscin in the retinal pigment epithelium in vivo. Autofluorescence was shown to be abnormally high in certain inherited diseases and low in the presence of retinal atrophy. In 1995 Delori et al $^{2}$ used a fundus spectro-phorometer to measure the autofluorescence in vivo at the fovea and at $7^{\circ}$ temporal to the fovea. Their results showed intrinsic fundus autofluorescence results from at least two fluorophores, a dominant fluorophore with peak emission at $620-630 \mathrm{~nm}$ (optimal excitation at 510 $\mathrm{nm}$ ), and a minor fluorophore with peak emission at 520 - $540 \mathrm{~nm}$ (optimal excitation at $430-470 \mathrm{~nm}$ ). They concluded from the spectral characteristics, correlation with age, topographic distribution and retinal location between the choriocapillaris and photoreceptors that fundus autofluorescence arises primarily from lipofuscin (dominant fluorophore) in the retinal pigment epithelium. The minor fluorophore is probably located in the neurosensory retina and could be flavin adenine dinucleotide (FAD) found in high concentrations in the mitochondria of the photoreceptor inner segments. Therefore, fundus autoflourescence primarily highlights lipofuscin which is a biomarker of ageing and oxidative cell damage ${ }^{3}$. Before discussing the technology it is important to understand the role of the outer retina (retinal pigment epithelium - photoreceptor complex) and the macular pigments in fundus autofluorescence photography.

\section{The retinal pigment epithelium (RPE)}

The retinal pigment epithelium is a single layer of cells, cuboidal in cross section and hexagonal when viewed from above. The zonula occludens bind the retinal pigment epithelial cells together forming a blood retinal barrier equivalent to the capillary endothelium of the retinal vasculature. The cells are roughly $10-14$ microns in size at the fovea but they become flatter and broader peripherally up to 60 microns in size. Overlying each retinal pigment epithelial cell are about 45 photoreceptor cells for which it is metabolically responsible ${ }^{4}$. The apical side 
facing the photoreceptors have long microvilli that reach up between and envelop the outer segments of the photoreceptors. Melanin pigment granules are concentrated in the apical end of the cells while the mid portion contains the nucleus, Golgi apparatus, endoplasmic reticulum, and digestive vesicles. The basal membrane has convoluted infolds to increase the surface area for the absorption and secretion of material.

The retinal pigment epithelium actively engages in oxidative metabolism: enzymes are synthesised for functions such as membrane transport, visual pigment metabolism, digestion of waste products as well as limiting the formation of free radicals which damage the lipid membranes. The retinal pigment epithelium also contributes to the function and maintenance of the inter receptor matrix which is critical to retinal adhesion. It is part of a complex system of cellular communication that controls vascular supply, permeability, growth and repair as well as other processes vital to retinal function ${ }^{5}$. The melanin pigment is present within cytoplasmic granules called melanosomes and is responsible for the absorption of stray light thus minimising light scatter. It also serves as a free radical stabiliser and can bind with toxins as well as retinotoxic drugs such as chloroquine and thioridazine.

Furthermore, the retinal pigment epithelium is responsible for cellular renewal by phagocytosis of the photoreceptor outer segment discs and the synthesis of new discs ${ }^{6}$. The phagocytosed discs become encapsulated in vesicles within the retinal pigment epithelial cells (phagosomes) which merge with lysozyme to digest the material. Fatty acids are retained and waste products are excreted across the basal retinal pigment epithelial membrane. Over time residual lipophilic lysosomal debris from unsaturated fatty acid oxidation contributes to the formation of lipofuscin. Lipofuscin molecules are not amenable to degradation by lysozymes and this lead to the accumulation of lipofuscin in the retinal pigment epithelial cells ${ }^{7}$. Lipofuscin accumulates gradually with age until the cells are severely clogged with the autofluorescent pigment with up to $19 \%$ of the cytoplasmic volume containing lipofuscin by the age of 80 years. The lipofuscin granules accumulate predominantly in the peripheral cell margin while the absorbing melanin granules are oriented toward the apical cell centre and the cell nucleus which is located at the basal cell side (see Figure 1). Retinal pigment epithelium cells also produce lipofuscin rich deposits that may accumulate as drusen within Bruch's membrane. As they enlarge drusen become the earliest lesions in age-related macular degeneration ${ }^{8}$.

It is not clear if lipofuscin damages retinal pigment epithelial cells or whether it is simply a marker for cellular damage, but excessive accumulation of retinal pigment epithelial lipofuscin in autosomal recessive Stargardt's macular degeneration is considered to be the cause of retinal pigment epithelium atrophy. In cultured retinal pigment epithelial cells exposed to blue light, lipofuscin induced apoptosis ${ }^{8}$.

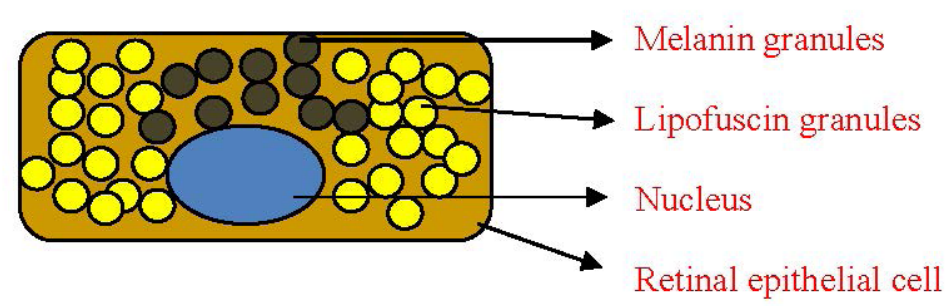

Figure 1: Diagram of ageing retinal pigment epithelial cell showing distribution of lipofuscin and melanin granules.

\section{Macular pigment}

The macular pigment consists of two carotenoids: lutein and zeaxanthin. Lutein is structurally related to $\alpha$-carotene and zeaxanthin to $\beta$-carotene; however, they are not provitamin A carotenoids. Lutein and zeaxanthin are especially concentrated in leafy green vegetables, many fruits and coloured vegetables such as squashes, sweet peppers, sweetcorn, and peas. In humans carotenoids cannot be synthesised de novo and have to be taken in by nutrition. Of all tissue, the retina has the highest concentration of xanthophyll almost exclusively lutein and zeaxanthin with the greatest agglomeration in the centre of the fovea. Zeaxanthin is the leading carotenoid in this location, declining with increasing eccentricity in favour of lutein ${ }^{8}$. The highest carotenoid concentration is in the pre-receptor axonal layer of the fovea and extra foveal macula. The macular pigment's absorbance spectrum peaks at $460 \mathrm{~nm}$, preventing damaging light to reach the photoreceptors (and retinal pigment epithelium cells) in the fovea.

The function of macular carotenoids is not 
clearly understood; still some functions can be extrapolated from their known biological, optical, and photochemical properties. These functions include blue light filtration, glare reduction, minimisation of chromatic aberration, improved fine detail discrimination, contrast enhancement as well as the maintenance of cellular health by reducing photo-oxidative damage of the neurosensory retina. Carotenoids are potent free radical scavengers and are particularly efficient in neutralising singlet oxygen. They are lipid soluble and it is therefore likely that they protect the polyunsaturated fatty acid rich membranes of the outer retina.

As stated before, lipofuscin accumulates in the retinal pigment epithelial cells and, when exposed to blue light, induces apoptosis in cultured retinal pigment epithelial cells ${ }^{8}$. When these cells are treated with lutein the phototoxic effect is greatly reduced. Lutein and zeaxanthin also reduce the amount of lipofuscin formed in cultured retinal pigment epithelial cells. It therefore appears as if macular pigment plays a major role in reducing the amount of lipofuscin formed, attenuating its phototoxic effects on the retinal pigment epithelial cells ${ }^{8}$. Eyes with agerelated macular degeneration had 38\% less lutein and zeaxanthin levels when compared to eyes of control subjects in the central $5^{\circ}$ of the macula ${ }^{8}$. Whether the loss of macular xanthophyll is an effect or contributory factor in the disease process are, however, not clear ${ }^{8}$.

The most commonly used non-evasive method to measure lutein and zeaxanthin levels in the macula in vivo is heterochromatic flicker photometry, a psychophysical technique where the patient seeks to eliminate flicker in a visual stimulus that alternates between two wavelengths: usually $460 \mathrm{~nm}$ blue light (maximum absorbance) and $540 \mathrm{~nm}$ green light (zero absorbance). The method depends on compliance and the condition of the macula. Fundus autofluorescence presents an option to easily and objectively measure the spatial distribution of macular pigment that requires less patient compliance and correlates well with psychophysical methods.

Delori et $a l^{9}$ pioneered a clinical routine using a modified scanning laser ophthalmoscope to measure the macular pigment density. The instrument was optimised to record autofluoresence images at $488 \mathrm{~nm}$ and $514 \mathrm{~nm}$. As mentioned previously, macular pigment absorption is high at $488 \mathrm{~nm}$ and practically zero at $514 \mathrm{~nm}$. Foveal and para-foveal autofluorescence recorded at the two wavelengths can be digitally subtracted to determine the macular pigment density as well as provide valuable information as regards disease processes affecting the macula. Rothenbueler ${ }^{10}$ studied the relationship between increased and decreased fundus autofluorescence signal and local macular pigment density in patients with age-related macular degeneration. They found that focal increases in fundus autofluorescence signal due to age-related macular degeneration did not lead to changes in local macular pigment densities, indicating that the layers of the retina containing macular pigment are not affected by the disease processes.

\section{Instrumentation}

Fundus autofluorescence can be imaged with a confocal scanning laser ophthalmoscope (cSLO) or a fundus camera. Both methods have to account for the natural fluorescence of the crystalline lens which interferes with and degrades the fundus autofluorescence signal.

\section{Fundus autofluorescence using the cSLO}

The autofluorescence intensity of the fundus is about two orders of intensity less than the background of a fluorescein angiogram at the most intense part of the dye transit. Camera systems with high sensitivity, image averaging capabilities and alignment to counter eye movements are required to capture fundus autofluorescence images with acceptable signal/noise ratios using safe retina exposure levels. Confocal scanning ophthalmoscopes optimally addressed these requirements as demonstrated by Von Rückmann ${ }^{1}$. Subsequently different scanning laser ophthalmoscopes (cSLOs) have been used and continuous technical innovations have resulted in higher quality fundus autofluorescence images ${ }^{11}$. Recently high resolution cSLOs and adaptive optics technology have led to high magnification imaging in which individual retinal pigment epithelial cells can be resolved in vivo ${ }^{12}$. Because the cSLO is confocal it can reject light emitted from the crystalline lens allowing good quality autofluorescent images. In order to reduce noise in the images, several scans must be taken and averaged which is then adjusted by 
the cSLO software. The images generally look good but makes consistent gray scale measurements of autofluorescence images impossible form one image to the next ${ }^{3}$.

Fundus autofluorescence can be excited between $430-600 \mathrm{~nm}$ but its highest efficiency is obtained with wavelengths from $430-510 \mathrm{~nm}$. Commercial SLO systems typically use an emission spectrum of $488 \mathrm{~nm}$ generated by an argon solid state laser. The emission spectrum is broad varying from $480 \mathrm{~nm}$ to $800 \mathrm{~nm}$ with maximum emission between $600 \mathrm{~nm}$ and $640 \mathrm{~nm}$ (lipofuscin maximal emission is between $620 \mathrm{~nm}$ and $630 \mathrm{~nm}$ ). A barrier filter inserted in front of the detector $(500,515$ or $521 \mathrm{~nm}$ depending on the cSLO used) eliminates reflected light as well as emissions below $521 \mathrm{~nm}$ allowing observation of the autofluorescence. Significant differences in image contrast, brightness and the range of gray scale levels which are important for image quality have been reported $^{13}$. These differences are important when comparing fundus autofluorescence images from different imaging devices.

Near infra-red fundus autofluorescence can be recorded using excitation wavelengths of 790 $\mathrm{nm}$ with emissions above $800 \mathrm{~nm}$ using a cSLO operated in indocyanine green mode. The signals are 60 - 100 times less intense than those obtained with short wavelength fundus autofluorescence but imaging is still possible although the contrast is low. Near infra-red fundus autofluorescence provides different information than short wavelength fundus autofluorescence. Its emission corresponds to areas of higher density melanin in the retinal pigment epithelium and choroidal pigment ${ }^{14}$.

Fundus autofluorescence using the fundus camera Although fundus cameras are capable of recording autofluorescent images, several factors influence the quality of the images. The flashlight of the fundus camera illuminates the entire posterior pole at the same time, resulting in a fluorescence signal derived from all tissue levels in the light beam reaching the CCD or CMOS chip. Fundus cameras are also more prone to light scattering and the crystalline lens autofluorescence around $488 \mathrm{~nm}$ reduces the image contrast tremendously ${ }^{3}$. The influence of the lens autofluorescence can be eliminated by moving the excitation and emission wavelengths for the fundus autofluorescence toward the red end of the spectrum thereby enhancing the contrast of the images.

The filters used with the fundus camera are called matched interference filters and was developed by Spaide $^{15}$ in 2003. The excitation filters' wavelength was chosen to be between 535 - $585 \mathrm{~nm}$ which is outside the absorption curve of fluorescein, making fundus autofluorescence imaging possible after fluorescein angiography unlike images obtained with the cSLO. The excitation wavelengths are not absorbed by the macular pigments. A barrier filter ${ }^{15}$ is used to allow passage of wavelengths starting at $615 \mathrm{~nm}$. The autofluorescence signal produced by the fundus camera using longer wavelength excitation and barrier filters would theoretically result in the detection of autofluorescence from a dissimilar complement of fluorophores. Macular pigment absorption in the central retina occurs to a much lesser extent with the fundus camera than the cSLO. A systematic comparison between the two imaging systems is still lacking ${ }^{3}$.

\section{Normal fundus autofluorescence image}

The characteristics of a normal fundus autofluorescence image include the following

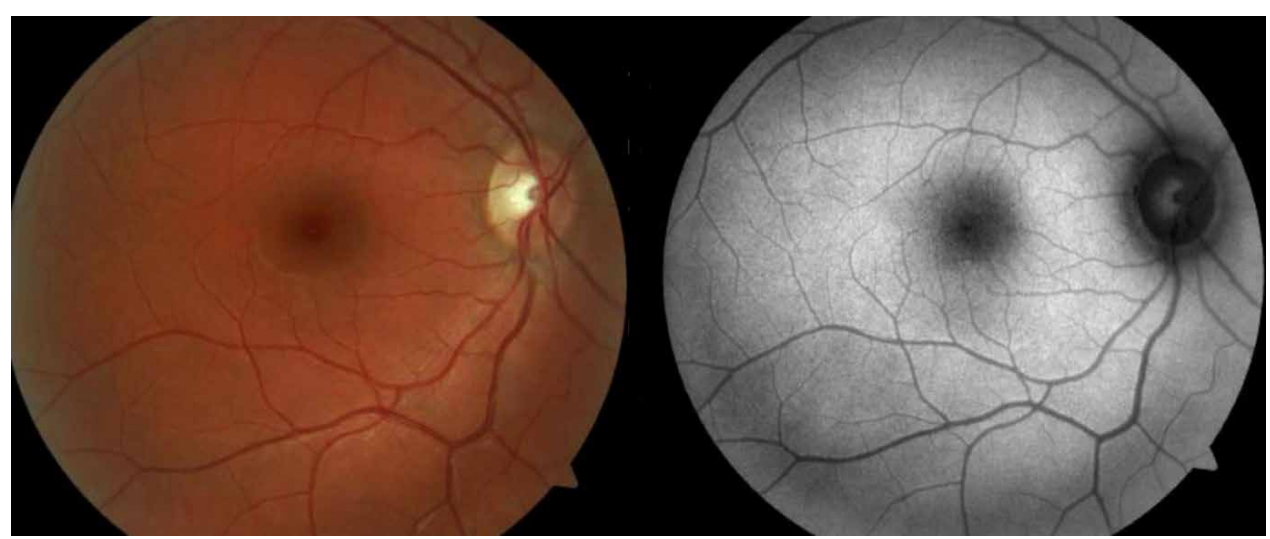

Figure 2: Conventional fundus and fundus autofluorescence photograph of a normal eye. Note dark macula, blood vessels and disc. (Photograph courtesy of Dr Peter Ambrose). 
features. The optic nerve head appears dark because of the absence of retinal pigment epithelium and therefore the fluorescent lipofuscin. The retinal vessels are associated with markedly reduced fundus autofluorescence due to the absorption phenomena from the blood contents. The fundus autofluorescence signal is markedly reduced at the foveal centre due to the macular pigment. The signal increases gradually as the macular pigment density decreases and then remains fairly constant throughout the rest of the fundus as shown in Figure 2.

Fundus autofluorescent signals can be reduced due to factors such as a reduction in retinal pigment epithelial lipofuscin density, increased retinal pigment epithelial melanin content, and absorption from extracellular material such as cells or fluid anterior to the retinal pigment epithelium. Fundus autofluorescent signals can be increased by factors such as excessive retinal pigment epithelial lipofuscin accumulation, lack of absorbing material, optic nerve head drusen, and artefacts ${ }^{16}$.

For the evaluation and interpretation of a fundus autofluorescent image of an individual patient it is essential to correlate the findings with funduscopy and other imaging techniques such as optical coherence tomography and fluorescein angiography. It is also recommended that a standardised protocol for image capturing is followed to improve image quality and facilitate the analyses of fundus autofluorescence images $^{3}$.

\section{Identification of early stages and pheno typing of retinal disease}

Lipofuscin accumulates in the retinal pigment epithelium in many macular and retinal dystrophies causing altered autofluorescence ${ }^{17}$. In vivo recording of lipofuscin accumulation may give important clues to the pathogenesis and progress of a number of diseases. Pale, yellow deposits are visible ophthalmoscopically at the level of Bruch's membrane and the retinal pigment epithelium in Best's disease; adult vitelliform macular dystrophy and Stargardt's macular dystrophy show markedly increased fundus autofluorescence intensities. These flecks are more clearly delineated with fundus autofluorescence images than with conventional fundus photography. However, a wide variation in clinical phenotypes can occur in patients with Stargardt's disease which may correlate with the different clinical types of the disease ${ }^{18}$.

Patients with age-related macular degeneration demonstrated focal accumulation of fluorescent lipofuscin related to focal increased areas of fundus autofluorescence and hyperpigmentation ${ }^{19}$. Von Rückmann et al ${ }^{20}$ corroborated the findings and suggested that fundus autofluorescence imaging can be used to document the metabolic activity of the retinal pigment epithelium in vivo. Fundus autofluorescence in patients with age-related macular degeneration demonstrating a patchy pattern indicates a higher risk of progression of the disease and subsequent vision loss ${ }^{21}$.

Fundus autofluorescence has a number of useful applications relating to the diagnosis of macular degeneration and other disease.

1. It is helpful in eliminating a number of similar looking diseases such as adult onset foveal macular dystrophy where material has been deposited beneath under, the retina. The appearance is similar to wet age-related macular degeneration with optical coherence tomography, but with fundus autofluorescence a tremendous amount of autofluorescence is present, which is not the case with standard macular degeneration.

2. Fundus autoflourescence is also useful to follow patients with geographic atrophy allowing accurate discrimination of the boundaries of atrophic patches. Increased rim autofluorescence is associated with an increased rate of expansion of geographic atrophy ${ }^{22}$.

3. Fundus autofluorescence can enhance our understanding of choroidal neovascularisation in patients with age-related macular degeneration and visual prognosis before treatment is initiated. Preserved autofluorescence in patients with recent onset choroidal neovascularisation indicates viable retinal pigment epithelium which has implications for visual prognosis ${ }^{23}$

4. Drusen visible with fundus photography may not correlate with notable fundus autofluorescence changes and likewise areas of increased fundus autofluorescence may not correspond with areas of hyperpigmentation or soft or hard drusen ${ }^{3}$. Meyerle et al ${ }^{24}$ compared fundus autofluorescence, conventional fundus photography, and fluorescein angiography in the detection of basal laminar drusen. Their findings 
demonstrated a higher sensitivity with fundus autofluorescence when compared to fundus photography. Moreover, when compared with fluorescein angiography, fundus autofluorescence also showed a higher sensitivity for the detection of basal laminar drusen. They concluded that fundus autofluorescence is an essential noninvasive clinical procedure in diagnosing and following basal laminar drusen and its sequelae ${ }^{24}$.

5. Fundus autofluorescence is useful in the differential diagnosis of choroidal nevi and melanomas. Autofluorescent patterns of choroidal melanocytic lesions are classified as either patchy (distinct areas of increased autofluorescence between areas of normal autofluorescence) or diffuse (increased autoflourescence with indistinct borders over a larger part of the lesion). Choroidal melanomas present with either a diffuse or patchy pattern whereas nevi only present with a patchy pattern of autofluorescence ${ }^{25}$. Near infrared fundus autofluorescence may provide more information about choroidal pigmented lesions by exploring the melanin distribution ${ }^{14}$. Natesh et al ${ }^{26}$ compared the clinical characteristics of fundus autofluorescence and optical coherence tomography / scanning laser ophthalmoscopy seen with choroidal metastatic tumours. They found that fundus autofluorescence best defined surface characteristics and tumour margins and that optical coherence tomography better demonstrated intraretinal findings such as atrophy, subretinal fluid as well as retinal pigment epithelium changes.

6. In acute central serous retinopathy an increase in autofluorescence appears to be a consistent finding which is not readily explained. It is possible that degradation of phagosomal material may be defective and this may result in an increase in autofluoresence. On the other hand, it may indicate higher metabolic activity. In chronic disease the demise of photoreceptors results in a mixed picture of reduced and focal increased autofluorescence due to the reduced metabolic activity in the retinal pigment epithelium ${ }^{27}$ as shown in Figure 3.

7. Fundus autofluorescence is also useful for the early detection of retinal changes associated with systemic use of chloroquine and hydroxychloroquine. Typically retinal pigment epithelial abnormalities begin with a fine

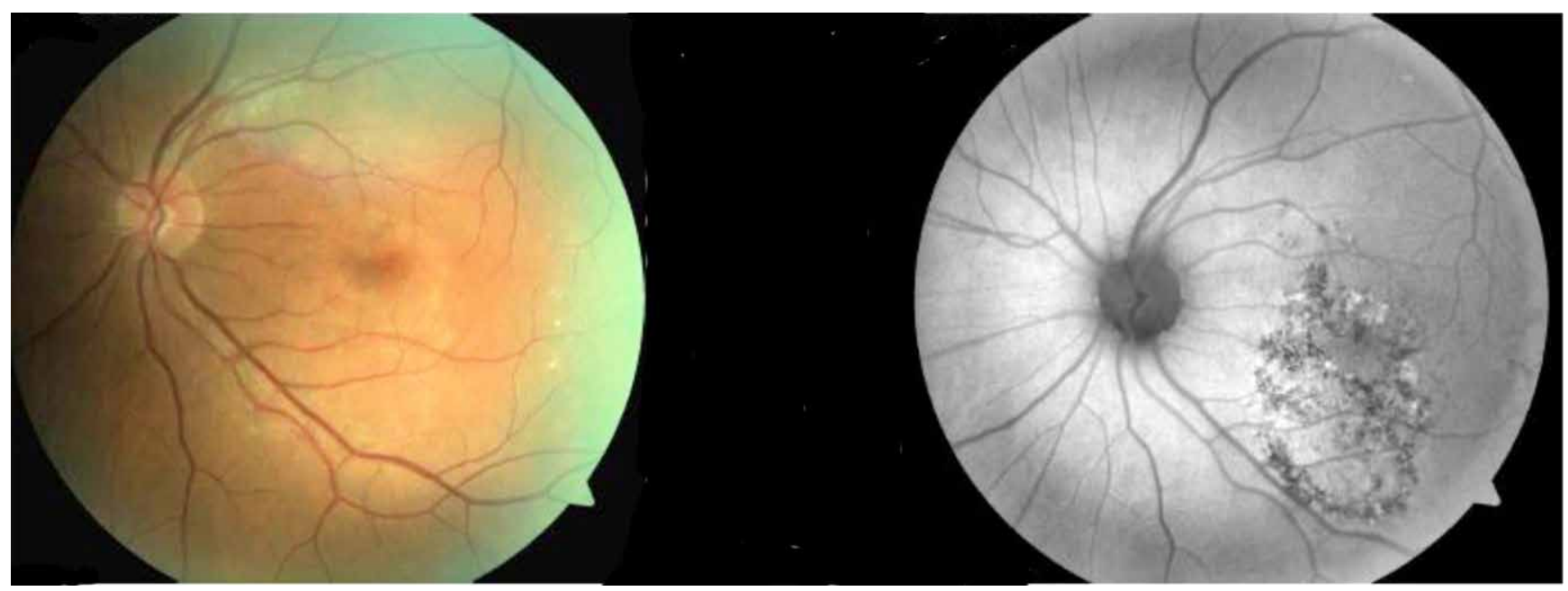

Figure 3: Central serous chorioretinopathy (CSR). An increase in autofluorescence is normally seen in acute CSR probably indicating an increased metabolic activity of the retinal pigment epithelium. The above lesion is probably long standing judging from the focal and decreased autofluorescence which would indicate reduced metabolic activity in the retinal pigment epithelium due to photoreceptor loss. (Photograph courtesy of Dr Peter Ambrose.) 


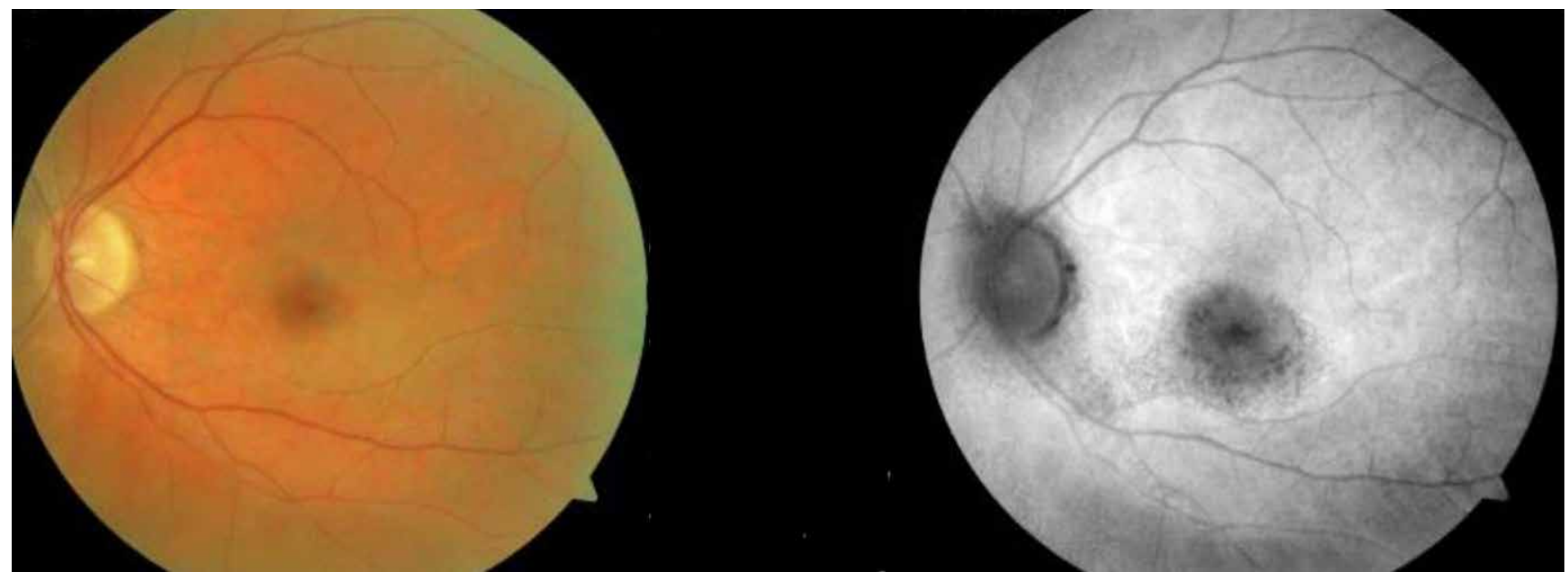

Figure 4: Chloroquine / hydroxychloroquine retinopathy. Retinal pigment epithelium abnormalities develop with long-term CQ/ HCQ intake. The abnormalities begin with a fine pericentral ring of increased autofluorescence which later widens. Firstly a mottled and then a general loss of the retinal pigment epithelium can be seen due to an absence of autofluorescence as shown in the above example. (Photograph courtesy of Dr Peter Ambrose.)

pericentral ring of increased autofluorescence which then widens: initially a mottled and later a general loss of retinal pigment epithelium is indicated by an absence of autofluorescence ${ }^{28}$ (see Figure 4).

\section{Conclusion}

Fundus autofluorescence provides a non-invasive, in vivo clinical index of retinal pigment epithelium lipofuscin and macular pigment density which can be used to enhance our understanding of retinal disease pathogenesis by:

- Tracking lipofuscin deposition and distribution in the retinal pigment epithelium,

- Diagnosing retinal disease before functional loss occurs,

- Identifying risk factors that may affect lipofuscin accumulation in the retinal pigment epithelium,

\section{References}

1. Von Rückmann A, Schmidt KG, Fitzke FW, Bird AC, Jacobi KW. Dynamics of accumulation and degradation of lipofuscin in retinal pigment epithelium in senile macular degeneration. Klin Monbl Augenheilkd 1998 213(1) 32-37.

2. Delori FC, Goger DG, Dorey CK. Age-related accumulation and spatial distribution of lipofuscin in RPE of normal subjects. Invest Ophthalmol Vis Sci 2001 42(8) 1855-1866.

3. Schmitz-Valckenberg S, Holz F. Fundus autofluorescence imaging: do you need it and when? Retinal Physician. http:// www.retinalphysician.com, 2008 Date accessed: 23/11/2010.

4. Hogan MJ, Alvarade JA, Weddell JE. Histology of the human eye. Philadephia: WB Saunders, 1971, 405-423.

5. Marmor MF. Anatomy and physiology of the retinal pigment epithelium. Ophthalmology 1999 8(2) 1-4.

6. Young RW. Visual cells and the concept of renewal. Inves Opthalmol 197615 700-725.

7. Sparrow JR, Wu Y, Nagasaki T, Yoon KD, Yamamoto K, Zhou J. Fundus autofluorescence and the bisretinoids of retina. Photochem Photobiol Sci 2010 9(11) 1480-1489.

8. Whitehead AJ, Mares JA, Danis RP. Macular pigment. Arch Ophthalmol 2006124 1038-1045.

9. Delori FC, Fleckner MR, Goger DG, Weiter JJ, Dorey CK. Autofluorescence distribution associated with drusen in age-related macular degeneration. Invest Ophthalmol Vis Sci 200041 496-504.

10. Rotenbuehler SP, Wolf-Schnurrbusch UE, Wolf S. Macular pigment density at the site of altered fundus autofluorescence. Graefes Arch Clin Experimental Ophthalmol 2011249 499. 504

11. Bindewald A, Jorzik JJ, Loesch A, Schutt F, Holz FG. Visualization of retinal pigment epithelial cells in vivo with digital high-resolution confocal scanning laser ophthalmoscopy. Am J Ophthalmol 2004137 556-558.

12. Bindewald A, Jorik JJ, Roth F, Holz FG. cSLO digital AF imaging. Ophthalmologe 2005102259.

13. Bellman C, Rubin GS, Kabanarou SA, Bird AC, Fitzke FW. Fundus autofluorescence imaging compared with different confocal scanning laser ophthalmoscopes. Br J Ophthalmol 200387 1381-1386.

14. Pilotto E, Ernesto AR, Midena E. OCT and autofluorescence: are they useful in the diagnosis of intraocular tumours? Retina Today 2009 71-74.

15. Spaide RF. Fundus autofluorescence and age-related 
macular degeneration. Ophthalmol 2003110 392-399.

16. Holz FG, Fleckenstein M, Schmitz-Valckenberg S, Bird AC. Evaluation of fundus autofluorescence images. Atlas of Fundus Autofluorescence Imaging. Berlin, Heidelberg: Springer-Verlag, 2007 chapter 8 71-77.

17. Boon CJ, Jeroen Klevering B, Keunen JE, Hoyng CB, Theelen T. Fundus autofluorescence imaging of retinal dystrophies. Vis Res 200848 2569-2577.

18. Dolar-Szczasny J, Mackiewicz J, Biziorek B, Zagórski Z. Fundus autofluorescence imaging in patients with Stargardt's dystrophy and fundus flavimaculatus. Klin Oczna 2007109 176-178.

19. Solbach U, Keilhauer C, Knabben H, Wolf S. Imaging of retinal autofluorescence in patients with age-related macular degeneration. Retina 199717 385-389.

20. Von Rückmann A, Fitzke FW, Bird AC. Distribution of pigment epithelium autofluorescence in retinal disease state recorded in vivo and its change over time. Graefes Arch Clin Exp Ophthalmol 1999237 1-9.

21. Einbock W, Moessner A, Schnurrgusch UE, Holz FG, Wolf S, FAM Study Group. Changes in fundus autofluorescence in patients with age-related maculopathy. Correlation to visual function: a prospective study. Graefes Arch Clin Exp Ophthalmol 2005243 300-305.

22. Fleckenstein M, Wolf-Schnurrbusch U, Wolf S, Von Strachwitz C, Holz FG, Schmitz-Valckenberg S. Imaging diagnostics of geographic atrophy. Ophthalmologe 2010 107 1007-1015.

23. Dandeker SS, Jenkins SA, Peto T. Autofluorescence imaging of choroidal neovascularisation due to age-related macular degeneration. Arch Ophthalmol 2005123 1507-1513.

24. Meyerle CB, Smith RT, Barbazetto IA, Yannuzzi LA. Autofluorescence of basal laminar drusen. Retina 200727 1101-1116.

25. Gündüz K, Pulido JS, Ezzat K, Salomao D, Hann C. Review of fundus autofluorescence in choroidal melanocytic lesions. Eye (London) 200923 497-503.

26. Natesh S, Chin KJ, Finger PT. Choroidal metastases; fundus autofluorescence imaging: correlation to clinical, OCT, and fluorescein angiographic findings. Ophthalmic Surg Lasers Imaging 20105 1-7.

27. Von Rückmann A, Fitzke FW, Fan J, Halfyard A, Bird AC. Abnormalities of fundus autofluorescence in central serous retinopathy. Am J Ophtalmol 2002133 780-786.

28. Kellner U, Renner AB, Tillack H. Fundus autoflourescence an mfERG for early detection of retinal alterations in patients using chloroquine/hydroxychloroquine. Invest Ophthal Vis Sci 200647 3531-3538. 